\title{
10. \\ Ueber die Convergenz einer nach den Sinussen und Cosinussen der Vielfachen eines Winkels fortschreitenden Reihe.
}

(Vom Herrn Professor Dirksen zu Berlin.)

1.

Bezeichnet $f(x)$ eine Function von $x$, einzig und allein an die Bedingung gebunden, dafs ihre Werthe innerhalb zwei gegebener Grenzen, z. B. von $x=-\alpha$ bis $x=+\alpha$, einschliefslich, wo $\alpha$ eine, übrigens beliebige, endliche Gröfse repräsentirt, beständig endlich und bestimmt bleiben, und nimmt man an, dafs es möglich sei, die Werthe dieser Function, innerhalb eben jener Grenzen ausschliefslich, durch eine Reihe von der Form:

$$
\begin{aligned}
& A_{\mathrm{o}}+A_{1} \cos \frac{\pi x}{\alpha}+A_{8} \cos \frac{2 \pi x}{\alpha}+A_{3} \cos \frac{3 \pi x}{\alpha}+\ldots A_{i} \cos \frac{i \pi x}{\alpha}+\text { etc. } \\
& +B_{2} \sin \frac{\pi x}{\alpha}+B_{2} \sin \frac{2 \pi x}{\alpha}+B_{3} \sin \frac{3 \pi x}{\alpha}+\ldots B_{i} \sin \frac{i \pi x}{\alpha}+\text { etc. }
\end{aligned}
$$

die, der Kürze wegen, dargestellt werden mag durch

$$
A_{0}+\sum_{x}^{\infty} A_{i} \cos \frac{i \pi x}{\alpha}+\sum_{x}^{\infty} B_{i} \sin \frac{i \pi x}{\alpha},
$$

auszudrücken, wo, ganz allgemein, $A_{i}$ und $B_{i}$ endliche und bestimmte, von $x$ unabhängige Grölsen bezeichnen: so lälst sich auf mehr als eine, und wohl am leichtesten auf die zuerst von Euler (Nova acta acad. Petrop. tom. XI. p. 116.), rücksichtlich eines verwandten Gegenstandes, mitgetheilte Weise zeigen, dafs alsdann sein mufs:

$$
\begin{gathered}
A_{0}=\frac{1}{2 \alpha} \int_{-a}^{+a} f(\mu) d \mu, \quad A_{i}=\frac{1}{\alpha} \int_{-a}^{+a} \cos \frac{i \pi \mu}{\alpha} \cdot f(\mu) d \mu, \\
B_{i}=\frac{1}{\alpha} \int_{-a}^{+\alpha} \sin \frac{i \pi \mu}{\alpha} \cdot f(\mu) d \mu,
\end{gathered}
$$

wo $\mu$ eine Hülfsgröfse, und $f(\mu)$ eine solche Function von $\mu$ bezeichnet, in welche $f(x)$ übergeht, indem man $x=\mu$ setzt.

Substituirt man diese Werthe für $A_{i}$ und $B_{i}$ in die obige Reihe, so entsteht die Gleichung:

(1.) $f(x)=\frac{1}{2 \alpha} \int_{-\alpha}^{+\alpha} f(\mu) d \mu+\frac{1}{\alpha} \sum_{x}^{\infty} \int_{-a}^{+a} \cos \frac{i \pi(x-\mu)}{\alpha} \cdot f(\mu) d \mu$, 
welche, in Verbindung mit den vorigen Bedingungen, ein sehr bekanntes Theorem ausspricht.

Man sieht aber leicht, dafs dieses Theorem durch die hier bezeichnete Betrachtungsweise, auf keinen Fall als begründet angesehen werden darf, in so fern nicht unter andern auch die Convergenz der auf der rechten Seite von (1.) befindlichen Reihe gesondert dargethan wird.

Zu beweisen, dafs eben diese Reihe, unter den obigen Bedingungen von $f(x)$ und $\alpha$, stets einen endlichen und bestimmten Werth hat, ist der nächste Gegenstand der folgenden Betrachtungen, bei denen, zur Ablürzung, der Buchstabe $E$, sowohl mit als ohne Ordnungszahl, als Repräsentant einer endlichen und bestimmten Grölse überhaupt, und das Zeichen $G_{z}$ vor einer Function von $z$, zur Andeutung desjenigen Werthes dienen wird, welchem sich der Werth der Function von $z$ ins Unbestimmte nähert, indefs der numerische Werth von $z$ als ins Unbestimmte zunehmend gedacht wird.

\section{2.}

Bezeichnet $\psi(\mu)$ eine continuirliche unbestimmte Function von $\mu$, von $\mu=\mu_{\mathrm{o}}$ bis $\mu=\mu_{1}$ einschliefslich, so hat man bekanntlich, mittelst partieller Integration, $\frac{d \psi(\mu)}{d \mu}=\psi^{\prime}(\mu)$ setzend:

$$
\begin{aligned}
& \int_{\mu_{0}}^{\mu_{\mathrm{x}}} \cos \frac{i \pi(x-\mu)}{\alpha} \psi(\mu) d \mu=\frac{\alpha}{i \pi}\left\{\sin \frac{i \pi\left(x-\mu_{\mathrm{x}}\right)}{\alpha} \psi\left(\mu_{1}\right)-\sin \frac{i \pi\left(x-\mu_{\mathrm{o}}\right)}{\alpha} \psi\left(\mu_{0}\right)\right\} \\
& \quad+\frac{\alpha}{i \pi} \int_{\mu_{\mathrm{o}}}^{\mu_{\mathrm{t}}} \sin \frac{i \pi(x-\mu)}{\alpha} \psi^{\prime}(\mu) d \mu ;
\end{aligned}
$$

(2.) $\left\{\begin{aligned} \sum_{\mathrm{x}}^{\infty} \int_{\mu_{\mathrm{o}}}^{\mu_{\mathrm{x}}} \cos \frac{l \pi(x-\mu)}{\alpha} \psi(\mu) d \mu= & -\frac{\alpha}{\pi} \psi\left(\mu_{1}\right) \sum_{1}^{\infty} \frac{1}{i} \sin \frac{i \pi\left(x-\mu_{\mathrm{x}}\right)}{\alpha} \\ & +\frac{\alpha}{\pi} \psi\left(\mu_{0}\right) \sum_{x}^{\infty} \frac{1}{i} \sin \frac{i \pi\left(x-\mu_{0}\right)}{\alpha} \\ & +\frac{\alpha}{\pi} \sum_{1}^{\infty} \int_{\mu_{0}}^{\mu_{\mathrm{x}}} \frac{1}{i} \sin \frac{i \pi(x-\mu)}{\alpha} \psi^{\prime}\left(\mu_{0}\right) d \mu,\end{aligned}\right.$ eine Gleichung, durch welche die Reihe (1.) auf drei andere zurückgeführt wird.

Betrachtet man nun die Reihe $\sum_{x}^{\infty} \frac{1}{i} \sin i z$, so ergiebt sich sehr leicht, dafs man hat, in so fern $n$ eine ganze Zahl bezeichnet:

$$
\sum_{z}^{\infty} \frac{1}{i} \sin i z=0 \text {, für } z= \pm 2 n \pi
$$

$=\frac{T}{2} \pi-\frac{1}{2} t$, für alle übrige Werthe von $z$, 
172 10. Dirksen, üb. d. Convergenz d. nach Sinussen u. s. w. fortschreitenden Reihe.

wo $t$ gegeben wird durch die Gleichung $z= \pm 2 n \pi+t, n$ so genommen gedacht, dafs der Werth von $t$ zwischen 0 und $+2 \pi$ falle.

Diesem nach hat man ganz allgemein

$$
\text { (3.) } \sum_{z}^{\infty} \frac{1}{i} \sin i z=E_{\rho} \text {, }
$$

Setzt man nun abwechselnd $z=\frac{i \pi\left(x-\mu_{1}\right)}{\alpha}, z=\frac{i \pi\left(x-\mu_{0}\right)}{\alpha}$, und überlegt zugleich, dafs man, der obigen Annahme nach, hat

so erlangt man

$$
\psi\left(\mu_{0}\right)=E_{0}, \quad \psi\left(\mu_{1}\right)=E_{1},
$$

$$
\text { (4.) }\left\{\begin{array}{l}
-\frac{\alpha}{\pi} \psi\left(\mu_{1}\right) \sum_{x}^{\infty} \frac{1}{i} \sin \frac{i \pi\left(x-\mu_{1}\right)}{\alpha} \\
+\frac{\alpha}{\pi} \psi\left(\mu_{0}\right) \sum_{x}^{\infty} \frac{1}{i} \sin \frac{i \pi\left(x-\mu_{0}\right)}{\alpha}
\end{array}\right\}=E_{2} .
$$

Ferner hat man, wie man leicht sieht,

$$
\begin{aligned}
\sum_{\mathrm{I}}^{\infty} \int_{\mu_{0}}^{\mu_{\mathrm{I}}} \frac{1}{i} \sin \frac{i \pi(x-\mu)}{\alpha} \psi^{\prime}(\mu) d \mu & =\int_{\mu_{0}}^{\mu_{\mathrm{x}}} \psi^{\prime}(\mu) d \mu G_{i} \sum_{\mathrm{I}}^{i} \frac{1}{i} \sin \frac{i \pi(x-\mu)}{\alpha} \\
& =\int_{\mu_{0}}^{\mu_{\mathrm{I}}} \psi^{\prime}(\mu) E_{\rho} d \mu \text { [nach (3.)]. }
\end{aligned}
$$

In so fern daher auch $\psi^{\prime}\left(\mu_{0}\right)$, innerhalb der Grenzen der Integration einschliefslich, beständig endlich und bestimmt bleibt, und $\mu_{0}, \mu_{1}$ ebenfalls endlich sind, hat.man bekanntlich

$$
\text { (5.) } \sum_{I}^{\infty} \int_{\mu_{0}}^{\mu_{1}} \sin \frac{i \pi(x-\mu)}{\alpha} \psi^{\prime}(\mu) d \mu=E_{3} \text {. }
$$

Verbindet man die Gleichung (4.) und (5.) mit (2.), so kommt, die vorigen Bedingungen als erfüllt betrachtend:

(6.) $\sum_{x}^{\infty} \int_{\mu_{0}}^{\mu_{x}} \cos \frac{i \pi(x-\mu)}{\alpha} \psi(\mu) d \mu=E_{2}+\frac{\alpha}{\pi} E_{3}=E_{4}$

3.

Die für $\psi^{\prime}(\mu)$ hervorgetretene Bedingung ist mit Bezug auf den Satz, in der Gleichung (6.), diese an und für sich betrachtet, enthalten, keine nothwendige, sondern lediglich eine, aus der besondern Betrachtungsweise hervorgegangene zufällige. Der Satz selbst behält seine volle Gültigkeit, wenn auch $\psi^{\prime}(\mu)$, innerhalb der Grenzen der Integration einschliefslich, ein-oder mehrmals unendlich wird, wofern nur $\psi(\mu)$ beständig endlich bleibt. Um die Begriffe festzustellen, wollen wir annehmen, dafs $\alpha$ eine, zwischen $\mu_{0}$ und $\mu_{2}$ enthaltene Grölse bezeichne, und dafs $\psi^{\prime}(\mu)$ lediglich für diesen Werth von $\mu$ unendlich werde. 
Da bekanntlich ganz allgemein

$$
\begin{aligned}
\sum_{\mathrm{I}}^{\infty} \int_{\mu_{0}}^{\mu_{\mathrm{I}}} \cos \frac{i \pi(x-\mu)}{\alpha} \psi(\mu) d \mu & =\sum_{\mathrm{I}}^{\infty} \int_{\mu_{0}}^{a-\frac{\mathrm{I}}{2} \theta} \cos \frac{i \pi(x-\mu)}{\alpha} \psi(\mu) d \mu \\
& +\sum_{\mathrm{I}}^{\infty} \int_{a-\frac{1}{2} \theta}^{a+\frac{1}{2} \theta} \cos \frac{i \pi(x-\mu)}{\alpha} \psi(\mu) d \mu \\
& +\sum_{x}^{\infty} \int_{a+\frac{1}{2} \theta}^{\mu_{\mathrm{I}}} \cos \frac{i \pi(x-\mu)}{\alpha} \psi(\mu) d \mu
\end{aligned}
$$

ist, so hat man, in Folge der Annahme und der Gleichung (6.), wofern nur der numerische Werth von $\theta$ nicht gleich 0 ist, wie man sich leicht überzeugt:

$$
\sum_{I}^{\infty} \int_{\mu_{0}}^{\mu_{x}} \cos \frac{i \pi(x-\mu)}{\alpha} \psi(\mu) d \mu=E_{5}+\sum_{x}^{\infty} \int_{a-\frac{1}{2} \theta}^{a+\frac{1}{2} \theta} \cos \frac{i \pi(x-\mu)}{\alpha} \psi(\mu) d \mu .
$$

Da der numerische Werth von $\theta$ nach Belieben klein genommen werden kann, so wird derselbe bekanntlich dergestalt gewählt werden können, dafs sich der Werth von $\psi(\mu)$, von $\mu_{i}=a-\frac{1}{2} \theta$ bis $\mu=\alpha+\frac{1}{2} \theta$, mittelst einer sehr rasch convergirenden, nach ganzen Potenzen von $\theta$ fortschreitenden Reihe darstellen lasse. Verbindet man nun mit dieser Bemerkung die Gleichung (6.), so ergiebt sich, wie man leicht übersieht, die Gleichung

$$
\sum_{x}^{\infty} \int_{a-\frac{1}{2} \theta}^{a+\frac{1}{2} \theta} \cos \frac{i \pi(x-\mu)}{\alpha} \psi(\mu) d \mu=E_{6},
$$

die, mit (\%) verbunden, liefert:

$$
\text { (8.) } \sum_{x}^{\infty} \int_{\mu_{0}}^{\mu_{x}} \cos \frac{i \pi(x-\mu)}{\alpha} \psi(\mu) d \mu=E_{7} \text {, }
$$

welche Gleichung also, wie leicht zu übersehen, statt findet, wofern nur $\psi(\mu)$, innerhalb der Grenzen der Integration einschliefslich, stets continuirlich ist, und $\mu_{0}$ und $\mu_{1}$ endliche Gröfsen bezeichnen. Dies vorausgesetzt, möge $f(\mu)$ eine Function von $\mu$ bezeichnen, deren Werthe gegeben werden:

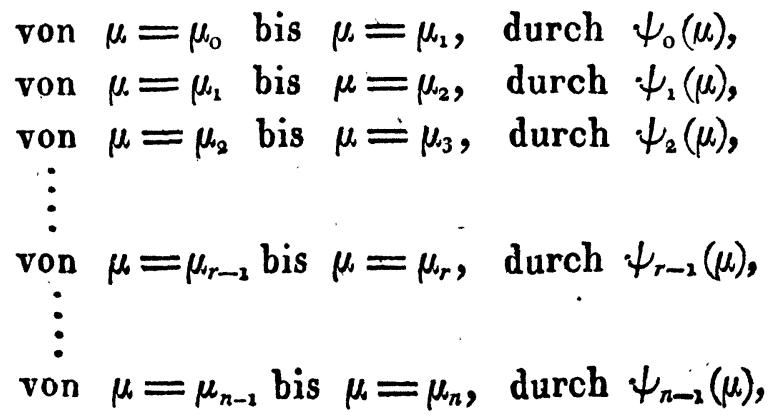


174 10. Dirksen, $\ddot{u} b$. d. Convergenz d. nach Sinussen u. s. w. fortschreitenden Reihen.

wo $\mu_{0}, \mu_{r}, \mu_{2}, \ldots \mu_{r}, \ldots . \mu_{n}$ entweder wachsend, oder abnehmend fortgehen, so hat man, in Folge von (8.):

folglich

$$
\sum_{1}^{\infty} \int_{\mu_{r-1}}^{\mu_{r}} \cos \frac{i \pi(x-\mu)}{\alpha} \psi_{r-1}(\mu) d \mu=E_{r-1},
$$

$$
\text { (9.) } \sum_{r=1}^{r=n} \sum_{1}^{\infty} \int_{\mu_{r-1}}^{\mu_{r}} \cos \frac{i \pi(x-\mu)}{\alpha} \psi_{r-1}(\mu) d \mu=\sum_{r=1}^{r=n} E_{r-1}=E \text {, }
$$

wofern nur ganz allgemein $\psi_{r-1}(\mu)$, von $\mu=\mu_{r-1}$ bis $\mu_{r}$ continuirlich ist, und $\mu_{r-1}$ und $\mu_{r}$ endliche GröIsen sind.

Setzt man nun

$$
\text { (10.) } \sum_{r=1}^{r=n} \sum_{1}^{\infty} \int_{\mu_{r-1}}^{\mu_{r}} \cos \frac{i \pi(x-\mu)}{\alpha} \psi_{r-1}(\mu) d \mu=\sum_{1}^{\infty} \int_{\mu_{0}}^{\mu_{n}} \cos \frac{i \pi(x-\mu)}{\alpha} f(\mu) d \mu \text {, }
$$

so erlangt man

$$
\text { (11.) } \sum_{1}^{\infty} \int_{\mu_{0}}^{\mu_{n}} \cos \frac{i \pi(x-\mu)}{\alpha} f(\mu) d \mu=E,
$$

wofern nur $f(\mu)$, innerhalb der Grenzen $\mu_{0}$ und $\mu_{n}$ einschliefslich, es sei übrigens continuirlich oder discontinuirlich, beständig endlich und bestimmt, und $\mu_{0}, \mu_{n}$ endliche Gröfsen repräsentiren.

Bezeichnet demnach $\alpha$ eine endliche Grölse, und setzt man

so kommt

$$
\mu_{0}=-\alpha \text { und } \mu_{s}=+\alpha \text {, }
$$

$$
\text { (12.) } \sum_{x}^{\infty} \int_{-a}^{+\alpha} \cos \frac{i \pi(x-\mu)}{a} \dot{f}(\mu) d \mu=E,
$$

wofern nur $f(x)$, von $x=-\alpha$ bis $x=+\alpha$ beständig endlich und bestimmt ist, welches Resultat von dem von Hrn. Cauchy (Mémoires de l'acad. d. scienc. an. 1823.) erhaltenen, in so fern verschieden ist, als letzteres bedingter zu sein scheint, und auch die mitunter geäufserte Ansicht, nach welcher ein Ausdruck von der Form (11.) und (12.), an und für sich betrachtet, nichts weiter als ein (bedeutungsloses) Symbol bildet, nicht bestätigt.

\section{4.}

Was die Begründung des Theorems durch die Gleichung (1.), in Verein mit den für $f(x)$ und $\alpha$ angegebenen Bedingungen ausgesprochen, anbelangt, so dürfte diese durch die in $\$ .1$. bezeichnete Betrachtung, in Verbindung mit dem Resultate von (12.), keinesweges als erledigt zu betrachten, und vielleicht auf folgende Weise am directesten zu bewerkstelligen sein. 
Bekanntlich ist:

$$
\left\{\begin{array}{c}
\sum_{1}^{\infty} \int_{\mu_{0}}^{\mu_{n}} \cos \frac{i \pi(x-\mu)}{\alpha} f(\mu) d \mu=G_{i} \int_{\mu_{0}}^{\mu_{n}} f(\mu) d \mu \sum_{2}^{i} \cos \frac{i \pi(x-\mu)}{\alpha} \\
=-\frac{\pi}{2} \int_{\mu_{0}}^{\mu_{n}} f(\mu) d \mu+\frac{\pi}{2} G_{i} \int_{\mu_{0}}^{\frac{\mu_{n}}{\sin \frac{\left(i+\frac{\pi}{2}\right) \pi(x-\mu)}{\alpha}}} \frac{\sin \frac{\frac{\pi}{2} \pi(x-\mu)}{\alpha}}{\alpha} f(\mu) d \mu,
\end{array}\right.
$$

und, nach (10.) und mittelst partieller Integration:

$$
\begin{aligned}
& \left(G_{i} \int_{\mu_{0}}^{\mu_{n}} \frac{\sin \frac{\left(i+\frac{x}{2}\right) \pi(x-\mu)}{\alpha}}{\sin \frac{\frac{\pi}{2} \pi(x-\mu)}{\alpha}} f(\mu) d \mu=-G_{i} \frac{1}{i+\frac{\pi}{2}} \sum_{r=1}^{r=n} \frac{\cos \frac{\left(i+\frac{x}{2}\right) \pi\left(x-\mu_{r}\right)}{\alpha}}{\sin \frac{\frac{\pi}{2} \pi\left(x-\mu_{r}\right)}{\alpha}} \psi_{r-2}\left(\mu_{r}\right)\right. \\
& +G_{i} \frac{1}{i+\frac{\pi}{2}} \sum_{r=1}^{r=n} \frac{\cos \frac{\left(i+\frac{x}{2}\right) \pi\left(x-\mu_{r-1}\right)}{\alpha}}{\sin \frac{\frac{1}{2} \pi\left(x-\mu_{r-1}\right)}{\alpha}} \psi_{r-2}\left(\mu_{r-1}\right) \\
& +G_{i} \frac{1}{i+\frac{x}{2}} \sum_{r=1}^{r=n} \int_{\mu_{r-1}}^{\mu_{r} \cos \frac{\left(i+\frac{x}{2}\right) \pi(x-\mu)}{\alpha}} \frac{\sin ^{\frac{\pi}{2} \pi(x-\mu)} \frac{1}{\alpha}}{\sum^{2}} d \psi_{r-1}(\mu) \\
& =0 \text {, }
\end{aligned}
$$

für alle Werthe von $x$, bei denen $\sin \frac{\frac{\pi}{2} \pi(x-\mu)}{\alpha}$, von $\mu=\mu_{0}$ bis $\mu=\mu_{n}$ einschliefslich, nicht Null, folglich $x-\mu$ nicht von der Form $\pm 2 n \propto$ wird. Obgleich dieses Resultat hier zunächst nur für den Fall hervortritt, wo $\frac{d \psi_{r-1}}{d \mu}(\mu)$, von $\mu=\mu_{r-1}$ bis $\mu=\mu_{r}$ einschliefslich, für alle Werthe von $r$, stets endlich bleibt, so überzeugt man sich dennoch auf die in §. 3. näher bezeichnete Weise sehr leicht, dafs solches auch dann noch allgemeine Gültigkeit hat, falls $\psi_{r-1}^{\prime}(\mu)$ ein-oder mehrmals unendlich werden sollte.

Erlangt aber $x-\mu$, für irgend einen $z$ wischen $\mu_{o}$ und $\mu_{n}$ einschliefslich enthaltenen Werth von $\mu$, einmal einen Werth von der Form $\pm 2 n \alpha$, wodurch alsdann $\mu= \pm 2 n \alpha+x$ wird; so hat man, da ganz allgemein

$$
\int_{\mu_{0}}^{\mu_{n}} \varphi^{\prime}(\mu) d \mu=\int_{\mu_{0}}^{ \pm 2 n \alpha+x-\theta} \varphi(\mu) d \mu+\int_{ \pm 2 n \alpha+x-\theta}^{ \pm 2 n \alpha+x} \int_{ \pm 2 n \alpha+x}^{ \pm(\mu) d \mu+} \int_{ \pm 2 n \alpha+x+\theta^{\prime}}^{ \pm(\mu) d \mu}+\int_{ \pm 2 n \alpha+x+\theta^{\prime}}^{\mu_{n}} \varphi^{ \pm}(\mu) d \mu
$$

ist, in Folge von (14.), wofern man zur Ablürzung 
setzt, wie leicht zu übersehen,

$$
\text { (15.) } \frac{\sin \frac{\left(i+\frac{x}{2}\right) \pi(x-\mu)}{\alpha}}{\sin \frac{\frac{x}{2} \pi(x-\mu)}{\alpha}} f(\mu)=\varphi(\mu)
$$

$$
\left(G_{i} \int_{\mu_{0}}^{\mu_{n}}(\mu) d \mu=G_{i} \int_{ \pm 2 n \alpha+x-\theta}^{ \pm 2 n \alpha+x}(\mu) d \mu+G_{i} \int_{ \pm 2 n \alpha+x}^{ \pm 2 n \alpha+x+\theta^{\prime}} \int_{ \pm}^{ \pm(\mu) d \mu,}\right.
$$

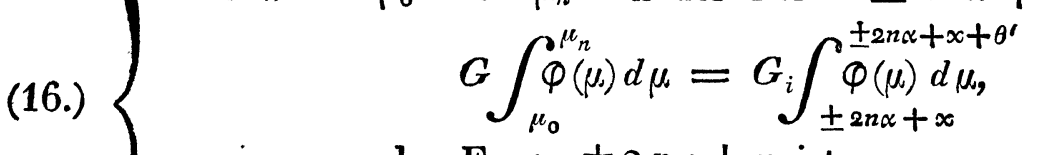

wenn $\mu_{0}$ von der Form $\pm 2 n \alpha+x$ ist;

$$
G_{i} \int_{\mu_{0}}^{\mu_{n}}(\mu) d \mu=G_{i} \int_{ \pm 2 n \alpha+x-\theta}^{ + \pm 2 n \alpha+x}
$$

wenn $\mu_{n}$ von der Form $\pm 2 n \phi+x$ ist;

wo $\theta$ und $\theta^{\prime}$ nach Belieben klein, jedoch nicht gleich Null genommen werden können.

Setzt man nun $\mu= \pm 2 n \alpha+x-\xi$, so kommt mit Rücksicht auf(15.):

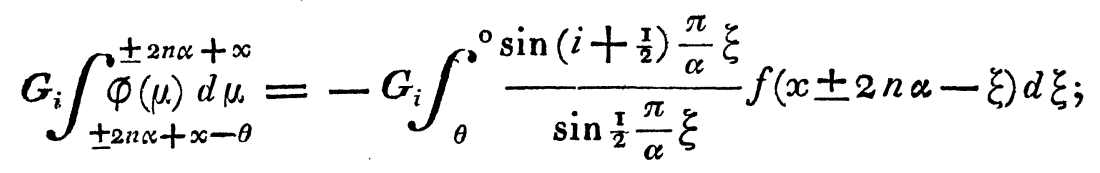

folglich, da $\theta$ nach Belieben klein genommen werden kann:

$$
G_{i} \int_{ \pm 2 n \alpha+x-\theta}^{ \pm(\mu n \alpha+x}=-2 f(x \pm 2 n \alpha) G_{i} \int_{\theta}^{0} \sin \frac{\left(i+\frac{1}{2}\right) \frac{\pi}{\alpha} \xi}{\frac{\pi}{\alpha} \xi} d \xi
$$

mithin, indem $\operatorname{man}\left(i+\frac{1}{2}\right) \frac{\pi}{\alpha} \xi=\zeta$ setzt :

$$
G_{i} \int_{ \pm 2 n \alpha+x-\theta}^{ \pm 2 n \alpha+x} \varphi(\mu) d \mu=+2 \frac{\alpha}{\pi} f(x \pm 2 n \alpha) \int_{0}^{\infty} \frac{\sin \zeta}{\zeta} d \zeta
$$

und endlich, da bekanntlich $\int_{0}^{\infty} \frac{\sin \zeta}{\zeta} d \zeta=\frac{\pi}{2} \pi$ ist :

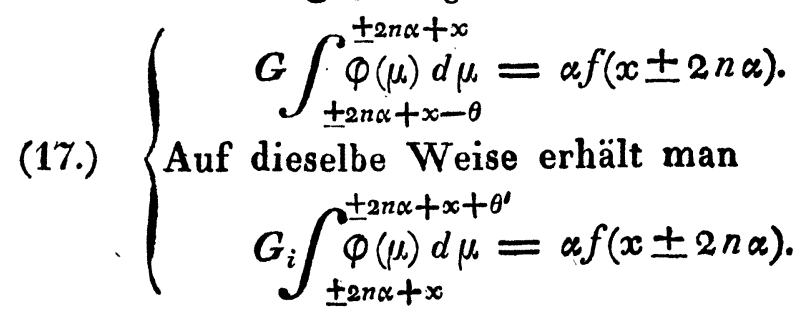


Fafst man nun die Resultate von (13.), (14.), (15.), (16.) und (17.) zusammen, so erlangt man folgenden allgemeinen Satz:

$$
\sum_{\mathrm{x}}^{\infty} \int_{\mu_{0}}^{\mu_{n}} \cos \frac{i \pi(x-\mu)}{\alpha} f(\mu) d \mu=-\frac{\mathrm{I}}{2} \int_{\mu_{0}}^{\mu_{n}} f(\mu) d \mu,
$$

wenn zwischen $\mu_{\mathrm{o}}$ und $\mu_{n}$ einschliefslich kein Werth für $\mu$ von der Form $\pm 2 n a+x$ enthalten ist;

(18.)

$$
\sum_{i}^{\infty} \int_{\mu_{0}}^{\mu_{n}} \cos \frac{i \pi(x-\mu)}{\alpha} f(\mu) d \mu=-\frac{1}{2} \int_{\mu_{0}}^{\mu_{n}} f(\mu) d \mu+\alpha f(x \pm 2 n \alpha) \text {, }
$$

wenn zwischen $\mu_{\mathrm{o}}$ und $\mu_{n}$ ausschliefslich ein Werth für $\mu$ von der Form $\pm 2 n a+x$ enthalten ist;

$$
\begin{aligned}
& \sum_{1}^{\infty} \int_{\mu_{0}}^{\mu_{n}} \cos \frac{i \pi(x-\mu)}{\alpha} f(\mu) d \mu=-\frac{1}{2} \int_{\mu_{0}}^{\mu_{n}} f(\mu) d \mu+\frac{1}{2} \alpha f(x \pm 2 n \alpha), \\
& \text { enn entweder } \mu_{o} \text { oder } \mu_{n} \text { von der Form } \pm 2 n \alpha+x \text { ist, }
\end{aligned}
$$

wo $n$ so genommen werden mufs, dafs $x \pm 2 n \propto$ zwischen $\mu_{0}$ und $\mu_{n}$ einschliefslich falle.

Ist aber zwischen $\mu_{0}$ und $\mu_{n}$ eine Anzahl von $(m+1)$ Werthen für $\mu$ von der Form $\pm 2 n \alpha+x$ vorhanden, von denen keiner mit den Grenzwerthen $\mu_{0}$ oder $\mu_{n}$ übereinstimmt, so findet man:

(19.) $\sum_{x}^{\infty} \int_{\mu_{0}}^{\mu_{n}} \cos \frac{i \pi(x-\mu)}{\alpha} f(\mu) d \mu=-\frac{1}{2} \int_{\mu_{0}}^{\mu_{n}} f(\mu) d \mu+\alpha \cdot \sum_{\rho=0}^{\rho=m} f\left[x \pm 2(n+\rho) \alpha_{0}\right)$.

5.

Setzt man nun, um sich von diesem allgemeineren Fall zu dem in Rede stehenden mehr besonderen zu wenden, $\mu_{0}=-\alpha, \mu_{n}=+\infty$, so übersieht man leicht, dafs es zu jedem Werthe von $x$ einen Werth, und auch nicht mehr als Einen Werth fur $\mu$, zwischen $-\alpha$ und $+\alpha$ enthalten, giebt, in Folge von welchem $x-\mu$ von der. Form $\pm 2 n \alpha$ wird.

Nach (18.) hat man also

$$
\left(\sum_{1}^{\infty} \int_{-a}^{+\alpha} \cos \frac{i \pi(x-\mu)}{\alpha} f(\mu) d \mu=-\frac{\pi}{2} \int_{-\alpha}^{+\alpha} f(\mu) d \mu+\alpha f(x \pm 2 n \alpha)\right. \text {, }
$$

für solche Werthe von $x$, für welche weder $x+\alpha$ noch $x-\infty$ (20.) $\{$ von der Form $\pm 2 n \alpha$ wird;

$$
\sum_{x}^{\infty} \int_{-\alpha} \cos \frac{i \pi(x-\mu)}{\alpha} f\left(\mu_{0}\right) d \mu=-\frac{1}{2} \int_{-\alpha}^{+\alpha} f(\mu) d \mu+\frac{1}{2} \alpha f(x \pm 2 n \alpha),
$$

für solche Werthe von $x$, für welche entweder $x+\alpha$ oder $x-\alpha$ von der Form $\pm 2 n \propto$ ist. 
178 10. Dirksen, üb. d. Convergenz d. nack Sinussen u. s. w. fortschreitenden Reihen.

Für alle diejenigen Werthe von $x$, welche zwischen $-\alpha$ und $+\infty$ enthalten sind, hat man offenbar $n=0$, und daher, indem man unter dieser Voraussetzung die Gleichungen (20.) nach $f(x)$ auflöst:

$$
\text { (21.) } f(x)=\frac{1}{2 \alpha} \int_{-\alpha}^{+\alpha} f(\mu) d \mu+\frac{1}{\alpha} \sum_{x}^{\infty} \int_{-\alpha}^{+\alpha} \cos \frac{i \pi(x-\mu)}{\alpha} f(\mu) d \mu \text {, }
$$

für alle Werthe von $x$, zwischen $-\alpha$ und $+\alpha$ ausschliefslich enthalten,

$$
\begin{gathered}
\text { (22.) } f(x)=\frac{1}{\alpha} \int_{-\alpha}^{+\alpha} f(\mu) d \mu+\frac{2}{\alpha} \sum_{x}^{\infty} \int_{-\alpha}^{+\alpha} \cos \frac{i \pi(x-\mu)}{a} f(\mu) d \mu, \\
\text { für } x=-\alpha \text { und } x=+\alpha,
\end{gathered}
$$

von denen (21.) mit der Gleichung (1.) einerlei ist.

Berlin, den 15. Januar 1829. 